\title{
Mariages sur la frontière du groupe national. Normalité et transgressions dans les unions matrimoniales libano-palestiniennes au Liban
}

Weddings on the boundary of the national group: norm and transgression in Lebanese-Palestinian marriages in Lebanon

Matrimonios en la frontera del grupo nacional. Normalidad y transgresiones en las uniones matrimoniales líbano-palestinas en Líbano

\section{Daniel MEIER}

\section{(2) OpenEdition}

Journals

\section{Édition électronique}

URL : http://journals.openedition.org/conflits/17347

DOI : $10.4000 /$ conflits. 17347

ISSN : $1777-5345$

Éditeur :

CCLS - Centre d'études sur les conflits lilberté et sécurité, L'Harmattan

\section{Édition imprimée}

Date de publication : 20 décembre 2008

Pagination : 31-44

ISBN : 1157-966 X

ISSN : 1157-996X

\section{Référence électronique}

Daniel MEIER, « Mariages sur la frontière du groupe national. Normalité et transgressions dans les unions matrimoniales libano-palestiniennes au Liban », Cultures \& Conflits [En ligne], 72 | hiver 2008, mis en ligne le 19 mai 2009, consulté le 30 mars 2021. URL : http://journals.openedition.org/conflits/ 17347 ; DOI : https://doi.org/10.4000/conflits. 17347 


\title{
Mariages sur la frontière du groupe national. Normalité et transgressions dans les unions matrimoniales libano-palestiniennes au Liban ${ }^{1}$
}

\author{
Daniel MEIER
}

Daniel Meier est docteur en sociologie politique, chargé d'enseignement à l'Institut de hautes études internationales et du développement à Genève. Il a co-dirigé avec Riccardo Bocco un numéro de la revue A contrario sur "La Palestine et les conflits $d u$ Moyen-Orient » et vient de publier Mariages et identité nationale au Liban (Karthala, 2008).

$\mathrm{D}$ epuis la fin de la guerre civile, la situation des réfugiés palestiniens au Liban a fait l'objet de nombreuses enquêtes et publications ${ }^{2}$. Il faut dire que, dans l'ensemble du monde arabe, le Liban se distingue par sa politique fort restrictive et discriminante à l'égard de ces « étrangers » dont le statut de réfugié n'est pas officiellement reconnu ${ }^{3}$. Les acteurs politiques de l'après-guerre ont donc érigé une véritable frontière entre Libanais et réfugiés Palestiniens au Liban. On peut même dire qu'une partie de l'identité nationale libanaise s'est reformulée autour de la figure honnie du réfugié palestinien, rendue responsable de la guerre civile que le Liban a connu entre 1975 et 1990 . De façon

1. L'auteur tient à remercier le Fonds national de la recherche scientifique suisse (FNS) pour son soutien, ainsi que les deux relecteurs anonymes pour leurs commentaires et suggestions.

2. Voir, par exemple, une des premières publications en français d'alors : al-Natour S., Les Palestiniens du Liban, Beyrouth, Dar al-Taqqadom al-Arabi, 1993. Parmi les récents travaux, voir Doraï M.K., Les Réfugiés palestiniens du Liban, Paris, CNRS, 2006.

3. Destremeau B., «Le statut juridique des Palestiniens vivant au Proche-Orient ", Revue d'études palestiniennes, $\mathrm{n}^{\circ} 48,1993$, pp. 35-62 ; Wadie S., “The Obligations of Host Countries to Refugees under International Law: The Case of Lebanon”, in Aruri N. (ed.), Palestinian Refugees. The Right of Return, London, Pluto Press, 2001, pp. 123-151.

4. Sur ce dernier point, voir Nassif Tar-Kovacs N., Les Rumeurs dans la guerre du Liban, Paris, CNRS, 1998. 
symptomatique, les travaux traitant de la réconciliation post-conflit ${ }^{5}$ ont largement laissé de côté la problématique des relations libano-palestiniennes, tandis que la politique de reconstruction / réconciliation mise en place par les gouvernements successifs de la seconde République a purement et simplement ignoré l'existence des réfugiés palestiniens ${ }^{6}$.

L'une des pistes permettant d'étudier la manière dont se délimite un groupe national par rapport à un groupe étranger consiste à se pencher sur leurs relations ${ }^{7}$, dans le cas qui nous intéresse les relations libano-palestiniennes ${ }^{8}$. Les liens matrimoniaux nous ont paru particulièrement intéressants en ce qu'ils permettent de voir et comprendre les délimitations de cette frontière symbolique, les conditions sociales de la transgression, la construction de l'acte matrimonial comme transgressif ou non transgressif en regard d'un contexte. En effet, de telles unions sont problématiques dans certains cas et pas dans d'autres, où elles peuvent même apparaître comme parfaitement banales. Cette fluctuation entre normalité et transgression qui semble caractériser ces mariages ouvre un champ de questions relatives aux facteurs explicatifs des divers degrés de transgression : comment expliquer pareils écarts dans la façon de vivre et penser de tels mariages ? Quels sont les points saillants de cette «frontière » invisible qui existe entre Libanais et réfugiés palestiniens du Liban ? Comment sont appréhendées ces unions matrimoniales au niveau des acteurs sociaux ? En somme, qu'est-ce que se marier signifie en pareil contexte?

Deux temps vont rythmer cette étude. Nous allons fournir quelques données de base sur le phénomène des unions matrimoniales libano-palestiniennes et cerner de façon générale les variables qui ressortent de l'enquête que nous avons menée ces dernières années. Ceci devrait permettre de saisir l'importance de la contextualisation des mariages pour appréhender la dimension transgressive qui peut leur être associée. Nous présenterons ensuite une série de profils et de témoignages d'acteurs des mariages libano-palestiniens, ces derniers présentant les registres argumentatifs mobilisés dans leur environnement pour qualifier ou disqualifier leur union matrimoniale, ainsi que les stratégies adoptées en fonction des difficultés rencontrées.

5. Huybrechts E., Douhayi C. (dirs.), «Reconstruction et réconciliation au Liban », Les Cabiers $d u$ Cermoc, n²3, 1999.

6. On en veut pour preuve le document officiel The Achievements 1993-1997, Damour, Ministry of Displaced, 1997.

7. Suivant en cela la fructueuse piste de Fredrik Barth, pour qui l'identité du groupe se construit précisément dans la relation avec l'Autre. Voir Barth F., "Boundaries and Connections", in Cohen A.P. (ed.), Signifying Identities. Anthropoligical Perspectives on Boundaries and Contested Values, London / New York, Routledge, 2000, pp. 17-36.

8. Voir Klaus D., Palestinian Refugees in Lebanon: Where to Belong?, Berlin, Klaus Schwarz Verlag, 2003. 


\section{Sphère matrimoniale et variables sociologiques}

L'enquête menée auprès des Libanais et Palestiniens qui ont franchi leur frontière nationale permet de cerner globalement quelles sont les variables susceptibles de transformer ce passage en action socialement proscrite, transgressive ou, à l'inverse, en acte relevant d'une certaine "normalité », au sens où il serait devenu / perçu comme banal ${ }^{9}$. Rappelons toutefois que, dans le Liban pluricommunautaire - dix-huit communautés religieuses y sont reconnues -, se marier n'a rien d'un acte ordinaire qui ne ferait qu'engager deux individus consentants. Il représente beaucoup plus que cela dans les pays méditerranéens en général où il engage l'ensemble de la famille élargie dans une relation d'alliance avec une autre famille, où se mêlent réputation et honneur et où le mariage signifie reconnaissance de l'autre groupe par le fait même de l'échange matrimonial ${ }^{10}$. Ceci se passe avec d'autant plus de difficultés que l'autre famille n'appartient pas au même groupe communautaire, au même groupe d'interconnaissance, parfois local, sans même parler du groupe national. Dans ces conditions, le mariage avec un individu d'un groupe étranger, qui plus est réfugié au Liban (avec lequel il y a eu d'importantes luttes politiques et militaires) a toutes les chances de voir son chemin semé d'embûches.

De façon plus générale encore, en l'absence de code civil, le mariage est soumis au Liban aux différents codes de statut personnel de chacune des communautés religieuses reconnues. Au-delà des problèmes qui peuvent s'ensuivrent - on pense notamment à l'interdiction formelle du mariage de la femme musulmane avec un homme non musulman - la subordination communautaire du fait matrimonial a été et reste un objet de controverse politique, notamment autour de l'impossibilité pour la femme libanaise de transmettre sa nationalité à ses enfants nés d'un père étranger, fussent-ils nés au Liban.

On voit ainsi que l'objet mariage constitue un enjeu pris entre sphère étatique et sphère religieuse. En effet, la rivalité entre ces deux univers est déjà ancienne autour de la définition même du mariage au Liban et autour de la possibilité d'y contracter un mariage civil ${ }^{11}$. Sans revenir sur les débats rela-

9. L’enquête a été conduite auprès d'une soixantaine de couples rencontrés entre 2001 et 2006. Pour plus de détails méthodologiques, nous nous permettons de renvoyer à : Meier D., Formes et frontières identitaires dans le Liban de l'après-guerre civile (1989-2005). Etude à partir des unions matrimoniales entre Libanais et Palestiniens, thèse de doctorat, Genève, Institut universitaire d'études du développement, 2006.

10. Voir Fox R., Anthropologie de la parenté. Une analyse de la consanguinité et de l'alliance, Paris, Gallimard, 1972 ; Davis J., People of the Mediterranean. An essay in Comparative Social Anthropology, London, Routledge \& Kegan Paul, 1977 ; Creswell R., « La parenté arabe au Liban : réflexions sur le modèle de l'histoire », in Breteau C.H., Zagnoli N. (dirs.), Production, pouvoir et parenté dans le monde méditerranéen, Paris, Geuthner, 1993, pp. 319-343.

11. Traboulsi I.A., "De Nicosie à Beyrouth : le mariage civil au Liban », Travaux et Jours, $\mathrm{n}^{\circ} 61$, 1998, pp. 53-63 ; Najm M.-C., " Pour une législation civile unifiée de la famille au Liban », Travaux et Jours, $\mathrm{n}^{\circ} 74,2004$, pp. 131-172. 
tifs à ce sujet, nous pouvons dire que le cadre administratif dans lequel se trouvent inscrites de telles unions est lui-même traversé par cette tension entre un pôle laïc et un pôle religieux, le second trouvant là le moyen de maintenir une influence déterminante sur les acteurs sociaux par le contrôle de leurs alliances matrimoniales. Gardien de la norme communautaire, le pôle religieux, via les codes de statut personnel, s'intègre dans l'économie politique des familles et tend à favoriser le maintien d'une ouverture très mesurée sur les autres communautés ${ }^{12}$. Cela ne manque pas de constituer une série d'entraves pour nombre d'unions « mixtes » sur le plan communautaire entre Libanais et Palestiniens.

Dans le cadre spécifique des unions matrimoniales avec des réfugiés palestiniens, l'organe administratif libanais relatif aux réfugiés - le Département des affaires palestiniennes, rattaché au ministère de l'Intérieur - doit préalablement donner son aval au mariage religieux. Un tel mariage aura des incidences diverses sur chacun des conjoints. En premier lieu, la femme palestinienne tire toujours un bénéfice de son mariage, puisqu'elle obtient un titre de séjour libanais et, après un an de mariage, le passeport libanais. A l'inverse, l'homme palestinien semble comparativement le « grand perdant » en cas de mariage avec une femme libanaise puisqu'il n'obtient aucun titre spécifique : il reste un réfugié palestinien. Le paradoxe veut que ce soit par l'alliance avec une femme étrangère vivant au Liban qu'il peut obtenir un titre de séjour lui permettant l'accès au marché du travail libanais.

Il faut en outre préciser que la procédure administrative concernant le mariage consacre la place subalterne de la femme dans le système libanais: d'une part, elle n'a pas à s'occuper de son statut administratif (c'est-à-dire qu'elle n'a aucune obligation d'annoncer son mariage), ce qui sous-entend qu'elle reste dépendante de l'organisation de ce dernier par des tiers, et notamment par son mari. En outre, comme on l'a relevé plus haut, elle ne peut transmettre la nationalité ni à son mari ni à ses enfants, ce qui montre bien le canal par lequel passe l'identité du groupe. Cela ajoute à la discrimination endurée par le groupe national palestinien dans la mesure où, en raison de sévères limitations dans l'accès à certaines professions, tout mariage d'une femme libanaise avec un réfugié palestinien implique que ses propres enfants ne pourront accéder aux mêmes métiers que les autres enfants de femmes libanaises, puisqu'ils auront été de facto étiquetés comme « réfugiés palestiniens » à leur naissance.

Notre enquête a permis d'élaborer différents profils d'acteurs ainsi que différentes étapes de l'histoire des relations libano-palestiniennes à partir de

12. Saadeh S., "Basic Issues Concerning the Personal Status Laws in Lebanon", in Scheffler T. (ed.), Religion between Violence and Reconciliation, Beirut, Ergon, 2002, pp. 449-456. 
trajectoires d'acteurs. Une proportion significative des couples interviewés a connu des difficultés à se marier, alors que d'autres n'ont même pas semblé s'apercevoir que de telles unions pouvaient poser problème. L'étude comparée de ces profils et des types de problèmes rencontrés nous permettent de dégager, à gros trait, quatre variables principales qui entrent en ligne de compte dans la compréhension des raisons de la construction d'une telle union matrimoniale en acte transgressif ou, a contrario, en acte anodin.

La variable communautaire semble la plus évidente à prendre en compte dans le contexte libanais. En réalité, elle n'est pas la plus significative et s'avère une des plus malléable : les acteurs sociaux en jouent, par delà les codes de statut personnel, composent des stratégies appropriées en fonction des sensibilités religieuses des familles de conjoints, et autorisent la conversion de l'un ou l'autre des époux, indépendamment du sexe et de la règle de la prééminence reconnue de l'appartenance communautaire de l'homme (et donc d'une préférence pour la conversion de la femme). Il faut toutefois ajouter que les mariages libano-palestiniens sont essentiellement, dans la période d'après-guerre civile, des mariages entre musulmans. En effet, les Palestiniens chrétiens ont été naturalisés en masse durant les années 1950. En outre, la guerre a opéré son effet de clivage, côté chrétien, si bien que « le Palestinien » y est plutôt perçu comme une figure repoussoir. On le constate, la variable communautaire n'est en soi pas signifiante, mais prend sens dans un contexte spécifique.

La géographie des mariages vient apporter un premier élément contextuel relativement consubstantiel à la variable communautaire, en raison de la forte territorialisation de cette dernière au cours de la guerre civile. Elle permet de constater une différence centrale entre le Sud (Saïda, Tyr) et le reste du pays, à l'exception de Beyrouth qui concentre à la fois des pratiques de proximité et d'éloignement entre Libanais et Palestiniens C'est en effet au Sud que l'on se marie le plus souvent et le plus facilement avec une personne issue du groupe national palestinien. En réalité, les acteurs sociaux vivent très souvent dans des régimes de proximité, d'interconnaissance ou de relations professionnelles. Cela s'explique avant tout par l'installation privilégiée des réfugiés dans la partie sud du Liban après la fuite de 1948, en raison de liens familiaux antérieurs, mais aussi dans la perspective d'un retour prochain en Palestine. Cette proximité reste teintée de méfiance tant chez les Libanais chiites que chez les Palestiniens, en raison des conflits qui ont opposé les deux groupes nationaux dans la région à l'époque de la guerre des camps (1985-1987) 13. Néanmoins, et de façon plus structurelle, la proximité contemporaine que l'on peut observer entre les deux groupes tire ses racines d'une histoire et d'une condition sociale communes. Ces deux variables mino-

13. Sur cet épisode, voir Abou Khalil A., "Shiites and Palestinians: Underlying Causes of the Amal-Palestinian Conflict", in Hagopian E.C. (ed), Amal and the Palestinians: Understanding the Battle of the Camps, Belmont, Arab-American University (Massachusetts), Papers $n^{\circ}$, 1985, pp. 9-13. 
rent ainsi la force de l'appartenance communautaire, celle-ci, comme à Tyr (ville chiite), n'étant plus un obstacle au mariage mais une identité en lutte pour une terre: "On est chiites et eux ils sont Palestiniens mais on se bat pour la même chose $14 »$. Enfin, cette variable géographique permet de rappeler une donnée fondamentale : environ la moitié des réfugiés palestiniens recensés par l'UNRWA (United Nations Relief and Works Agency, l'Office de secours et de travaux des Nations unies pour les réfugiés de Palestine dans le Proche-Orient) au Liban vit hors des camps ${ }^{15}$. Cela permet d'ajouter que les mariages sont un phénomène qui se déroule et s'actualise hors des camps plutôt que dans les camps. Cependant, la fréquentation d'un même emploi et de mêmes lieux d'interactions sont vecteurs de liens indéniables.

Ces liens sont justement tissés en raison du poids d'une variable connexe à celle de la géographie, que l'on pourrait nommer «capital économique ${ }^{16}$ ». Selon des études comparées en Méditerranée, cette variable influe au premier chef sur le champ des possibles du mariage; et l'on parle non pas de mariage de «classes ", mais de mariage "entre soi », dans des espaces sociaux relationnels où les référentiels sont partagés et où ils présentent une grande homogénéité socio-économique. C'est en ce sens que la géographie devient un facteur éclairant, dans la mesure où elle permet de cerner un aspect relationnel inhérent à la possibilité des unions matrimoniales : plus on est proche géographiquement et socialement des autres, plus on se marie avec eux. L'exemple de Saïda est assez parlant. Cette ville sunnite possède, outre les camps de Miyé ou Miyé et d'Ain el-Héloué, de nombreuses interpénétrations libano-palestiniennes dans son tissu urbain, et notamment dans la vieille ville. On s'y marie avec une grande facilité en raison d'une forte proximité qui a permis, avec le temps, l'établissement de liens de confiance, de réciprocité (y compris dans les mariages : il arrive qu'à la seconde génération, le groupe palestinien « rende » une fille au groupe libanais qui lui en avait « donné » une à la génération précédente).

Ce trait sociologique ne vaut toutefois que lorsque s'établit justement ce rapport de confiance et que la réputation de l'autre groupe familial est avérée. Or, seule la variable temporelle permet d'actualiser ce lien particulier au point de permettre l'échange matrimonial. Plus largement, c'est bien de l'histoire que les deux groupes nationaux partagent qu'il s'agit ici. A la fois la géographie, les rapports intercommunautaires et les situations socio-économiques subissent l'effet du temps et des changements qui leur sont associés. Cette quatrième variable attire l'attention du chercheur sur un ensemble plus vaste, celui des périodes historiques dans les relations libano-palestiniennes ${ }^{17}$. L'après-guerre

14. Formule récurrente entendue lors de divers entretiens à Tyr de 2002 à 2004.

15. Voir: www.un.org/unrwa

16. Bourdieu P., La Distinction, Paris, Minuit, 1979.

17. Sayigh R., Too Many Enemies: The Palestinians Experience in Lebanon, London, Zed Book, 1994. 
civile y apparait comme une ère marquée par les tensions intercommunautaires, contrastant avec une phase beaucoup plus ouverte aux échanges matrimoniaux au début des années 1970, qui a pris fin avec l'invasion israélienne de 1982 laquelle a mis à mal l'édifice politique palestinien au Liban. Elle se distingue tout autant de la période précédant les accords du Caire (1969), lesquels ont ouvert cet « âge d'or » des Palestiniens au Liban. En effet, entre 1948 et 1969, les réfugiés ont été contrôlés, parqués, surveillés et, de manière générale, largement stigmatisés (y compris les Palestiniens chrétiens et ce, malgré leur naturalisation). La variable temporelle permet en somme de mettre en relation chacune de ces époques avec la perception des mariages libano-palestiniens en faisant ressortir une tendance : plus les Palestiniens ont été puissants et plus les unions matrimoniales avec les Libanais sont apparues comme possibles. A l'inverse, plus ils se sont vus affaiblis et plus les mariages ont été perçus comme transgressifs et sont devenus difficiles.

Cette généralisation est évidemment encore trop problématique et nécessite des pondérations à la fois dans l'espace (différences entre régions du Liban) et dans le temps (les processus sociaux s'adaptant aux processus politiques de façon progressive). En outre, ces processus sociaux matrimoniaux, s'ils restent difficilement quantifiables faute de statistiques ou de recensements, se déployent le long d'un continuum entre transgression et normalité. Aussi, pour aller plus dans le détail, il nous paraît utile de saisir des trajectoires et propos d'acteurs dans leur contexte temporel et social. Ne pouvant se réduire à des systèmes ouverts ou fermés, les dimensions de ce qui est admis et de ce qui est tabou se présentent comme autant de jeux à la frontière où chaque norme semble gagner en plasticité et en relativité.

\section{Jeux à la frontière : entre braconnage et transgressions}

Afin de rompre avec la logique introduite par les variables et, en quelque sorte, reprendre le propos de cet article à partir du terrain, nous voudrions maintenant répertorier les genres de transgressions observées en les articulant avec les types d'unions matrimoniales. Ces réactions peuvent être ordonnées selon le degré d'intensité de la réaction qu'ils provoquent au sein des membres du groupe parental qui s'oppose à la décision du fils ou de la fille qui souhaite se marier.

\section{Les non-transgressions}

On trouve tout d'abord les unions libano-palestiniennes qui ne sont pas perçues comme transgressives. Bien qu'elles ne se limitent pas au groupe communautaire, elles n'engendrent pas de crise ou de conflit au sein des familles qui y sont engagées. Dans les profils à faibles revenus, ce type d'union est relativement commun, dans la mesure où il est basé sur une proximité sociale et économique, voire idéologique, avec l'autre. Le schéma, très fréquent, décrit 
une relation de voisinage et d'interconnaissance, comme le raconte Fouad, le mari palestinien d'une Libanaise, sunnite comme lui : "Je l'ai vu pendant un certain temps passer devant chez moi [...] j'en ai parlé avec mes parents et eux se sont renseignés et on a été faire la demande en mariage à son père dont la maison est dans le quartier $[\ldots]^{18} »$.

Pour d'autres profils de couples, ces unions, qu'elles aient lieu entre chrétiens ou entre musulmans, que ceux-ci soient peu ou très aisés, génèrent des discussions, concertations ou commentaires. Deux exemples peuvent illustrer ce point.

Fadwa est libanaise, veuve de 58 ans. Elle explique comment s'est passée sa rencontre avec son mari, un Palestinien, chrétien orthodoxe comme elle :

« Il connaissait mon père à Damas du fait du mouvement orthodoxe auquel il participait et il connaissait aussi ma sœur car elle avait envie de devenir bonne sœur [...] donc on s'était vu quand j'étais toute jeune, car lui il avait 17 ans de plus que moi [...]. Lui voulait devenir prêtre, mais il avait des problèmes d'argent alors il a changé d'avis... On se connaissait bien, on était des amis et... Je ne m'attendais pas du tout à ce qu'il me demande en mariage. [...] Quand c'est arrivé, en 1968, [...] j'ai demandé un délai de réflexion et je suis partie à Londres consulter mon frère et ma mère. Et on l'a fait venir pour que mon frère le connaisse. Et puis on s'est fiancés immédiatement après et trois mois plus tard on était marié 19. »

De son côté, Ahmad, un Libanais de 57 ans qui a fait carrière dans le monde universitaire à Beyrouth y a retrouvé, lors de son doctorat, une fille de famille palestinienne originaire du même village du Sud-Liban que lui. Ils sont tous deux sunnites.

« Mes parents connaissaient sa famille et on venait du même village alors... En tous cas, tout le monde était content dans nos familles. Bon... Au village, vous savez comment c'est... Il y a eu des gens qui ont dit qu'un type comme moi aurait dû épouser une Libanaise du village mais pas une Palestinienne ${ }^{20}$.

A ce niveau, le mariage avec un conjoint palestinien rejoint tous les autres mariages communs ou dits «normaux », c'est-à-dire relativement attendu : au Liban c'est une affaire sociale et, en conséquence, il est toujours question de savoir qui est le ou la promise et qui est sa famille. En un mot, il n'y a rien d'exceptionnel dans les actes ou commentaires relevés qui puisse permettre de

18. Entretien effectué à Saïda en septembre 2003.

19. Entretien effectué à Beyrouth en novembre 2003.

20. Entretien effectué à Beyrouth en septembre 2001. 
parler de "transgression ", au sens où cela poserait un problème pour les familles investies dans ces unions et, par voie de conséquence, pour les acteurs des couples souhaitant se marier.

\section{Les transgressions à impact réel}

Il y a ensuite des transgressions plus importantes, qui heurtent plus frontalement les normes sociales ou les représentations des membres de la parenté. Cellesci sont en général le fait de couples de communautés et parfois de religions différentes. Elles sont caractérisées par un temps d'attente entre le moment où le jeune couple voudrait convoler et le moment où ils reçoivent l'autorisation de le faire. Ce laps de temps est celui de la négociation, de la démonstration de la solidité et du sérieux de la demande du couple, voire des éventuels chantages. Les actions entreprises par les acteurs, dans cette catégorie de transgressions, ne varient pas forcément beaucoup par rapport à la catégorie suivante où l'on observe des ruptures. Les parents ou la famille investie dans l'affaire font en réalité preuve d'adaptation, et c'est bien toute la diversité de ces réactions qui fait la différence.

Dans le cas de Rada, une Libanaise chiite de 45 ans, épouser son ami palestinien rencontré sur les bancs de l'université n'a pas été chose aisée, moins en raison de la nationalité de ce dernier qu'à cause de la différence communautaire existante qui, traditionnellement, implique que la femme se convertisse à la confession de son mari :

« Mes parents n'acceptaient pas que je veuille épouser un homme parce que je l'aime... Si vous voulez, simplement le fait de dire que je l'aimais était contraire à leur conception [...]. Alors ils ont refusé notre projet de mariage et m'ont même menacée de ne plus me voir si je me mariais avec lui. [...] J'ai dû attendre que le temps passe et qu'ils comprennent que je ne changerais pas d'avis sans pour autant vouloir renoncer à ma famille. Enfin, ils ont accepté après un an et demi, mais ils ont mis la condition que ce soit un mariage chiite. Mon mari a été d'accord et on a pu se marier ${ }^{21 . ~ » ~}$

Dans le cas de Jean, un Libanais chrétien de 55 ans, la situation était un peu différente puisqu'il s'est marié civilement en France avec sa femme, une musulmane (sunnite) palestinienne, à Paris où ils vivaient en exil durant les années 1980. Néanmoins, respectant certains rites, ce laïc convaincu a attendu d'obtenir le consentement de son beau-père :

«On s'est mariés au bout de quatre ans avec ma femme. Son père était contre. [...] Cela dit, j'ai de l'estime pour lui, c'est un type vrai- 
ment intéressant. [...] En fait, les autres frères et sœurs de ma femme se sont aussi mariés de façon mixte, l'aîné de ses frères avait fait un mariage avec une chrétienne libanaise. Alors bon, pour nous... Sa mère était pour, mais le père était contre. Il préférait que ce soit un Palestinien. Et bien sûr, avant tout, que ce soit un musulman ! Finalement on s'est arrangé... Et ils ont accepté le mariage. Il faut dire qu'ils sont laïcs, alors ça aide aussi. ${ }^{22}$ »

\section{Les transgressions à ruptures}

Enfin, les transgressions les plus fortes sont celles qui occasionnent des ruptures plus ou moins longues entre parents et enfants. Elles se produisent au sein de groupes sociaux variés, mais tendanciellement moins dotés en capitaux économiques et culturels que dans les cas du groupe précédemment évoqué. Toutefois, ils présentent des franchissements du licite qui ne sont pas plus importants que ceux du groupe précédent. C'est donc bien l'ampleur de la réaction qui qualifie l'acte transgressif.

Yahyah ${ }^{23}, 32$ ans, palestinien clandestin au Liban ${ }^{24}$, raconte combien il a été difficile pour lui d'obtenir la main de celle qu'il a rencontrée à la faculté de psychologie, une Libanaise, sunnite comme lui.

«Au bout d'un an, quand on s'est mieux connus, je suis allé chez son père faire la demande en mariage. Il a refusé. Parce que j'étais palestinien et qu'il avait peur que je rentre en Palestine et que j'y emmène sa fille. C'était le seul problème. [...] Ma femme et moi, on a insisté et ça a duré cinq ans. »

Après une tentative avortée de rapt ou d'enlèvement (al-khatef) effectué avec le consentement de la fille ${ }^{25}$, le père s'élève contre Yahyah, lequel s'obstine. Menacé de mort au téléphone par son futur beau-père, il décide de l'affronter directement en se présentant le jour même devant lui. Le beau-père cède, et l'union peut avoir lieu. Toutefois, il tarde à reconnaître la légitimité du couple après le mariage et refuse de recevoir sa fille et son beau-fils. Il finit par s'y résoudre après la naissance du premier enfant.

22. Entretien effectué à Beyrouth en septembre 2001.

23. Entretien effectué à Beyrouth en novembre 2003.

24. Cette situation est le produit d'une réglementation restrictive qui dénie à un Palestinien le titre de réfugié et les droits qui lui sont associés par l'UNRWA et/ou par un gouvernement d'un pays où il séjourne. Voir Frontiers Association, Falling through the Cracks. Legal and Practical Gaps in Palestinian Refugee Status. A Case Study of Unrecognized Refugees in Lebanon, Beyrouth, 2005. Disponible sur : www.frontiersassociation.org

25. Pitt-Rivers J., " Mariage par rapt », in Peristiany J., Handman M.-E. (dirs.), Le Prix de l'alliance en Méditerranée, Paris, CNRS, 1989, pp. 53-71. 
Dans un autre cas, le mariage par enlèvement a réussi, comme le raconte Tarek ${ }^{26}$, le jeune mari libanais de Sabrin, tous deux sunnites, en présence des membres de la famille de cette dernière. Une des conséquences automatique d'un tel rapt est l'obligation du mariage qui en découle en raison de l'honneur perdu de la fille (une relation sexuelle réelle ou non, mais implicitement induite ayant pu avoir lieu à la faveur du rapt): «Dans ce mariage, le problème... était que... [très gêné] on s'est aimés très fort tout de suite alors on a voulu se marier... [dans un murmure] alors, j'ai enlevé Sabrin ». La mère de Sabrin intervient: "Moi j'étais pas d'accord que ma fille se marie... ». Son mari précise: "Nous voulions surtout qu'elle termine ses études, nous sommes très attentifs à cela, c'est précieux. Donc vous voyez, ce n'est pas parce qu'il est libanais que nous n'avons pas été d'accord». La sœur de Tarek ajoute : «Moi aussi, je lui ai dit que j'étais contre son mariage car il me semblait plus prudent d'attendre qu'il ait fini son armée ».

La résolution du problème, semble-t-il «à l'amiable », de cette rupture des conventions sociales due à cet enlèvement, lui-même conséquence d'un refus parental mais aussi et probablement d'une relation hors mariage, a pu avoir lieu en raison de la grande pauvreté du milieu dans lequel la famille de Sabrin évolue et la perspective d'une situation professionnelle stable promise à Tarek une fois son devoir militaire accompli.

Dans d'autres cas, la rupture entre parents et enfants semble beaucoup plus importante, comme pour Jamal ${ }^{27}$, libanaise druze de trente ans 28 devenue sunnite pour pouvoir épouser son ami Nabil après quatre ans de tractations infructueuses avec ses propres parents :

«Le problème, c'est seulement autour de la religion : mes parents auraient voulu pour moi un mari druze. Et le fait qu'il soit palestinien les a effrayés à cause des enfants qui seront automatiquement palestiniens. Pour moi ça m'est égal, ça ne fait rien. Bon... Alors... On s'est mariés sans personne de ma famille, vous voyez sur les photos... C'est la famille de Nidal. [...] Maintenant ça va mieux, mes parents sont ouverts... Bon pas autant que... Mais ils ont accepté le mariage. »

26. Entretien effectué à Saïda en septembre 2002. Cette catégorie de mariage «par rapt » théorisée par des anthropologues, comporte une dimension où, même si la jeune fille s'enfuit de son plein gré avec son prétendant, c'est lui qui assume «le rapt », la jeune fille restant dans une position passive. On mentionnera alors son consentement pour faire la différence avec le rapt sans consentement aux fins de mariage (qui arrive plus rarement).

27 . Entretien effectué à Beyrouth en septembre 2002.

28 . Il convient de préciser ici la spécificité du groupe druze en regard notamment de sa pratique matrimoniale assez largement endogame. Voir Abu Izzeddin N.M., The Druzes: A New Study of their History, Faith and Society, Leiden, E.J. Brill, 1993 ; Lallier F., «Liban : l'identité de la communauté druze du Chouf », Maghreb-Machrek, n¹65, 1999, pp. 3-15. 
Ces genres de transgressions nous montrent que ce n'est donc pas l'objet - l'acte de se marier avec quelqu'un de telle confession et de tel niveau social - qui, en soi, constitue un problème. C'est la perception construite par les acteurs de cette situation en tant que situation franchissant des limites que le groupe se donne et constituant un problème plus ou moins important qui est en jeu. A nos yeux, ces cas mettent en évidence aussi bien la dimension agissante que la dimension agie des acteurs 29 face aux normes et aux valeurs: ils s'avèrent capables de trouver des moyens de normaliser ce qui ne l'était pas, mais sont, dans le même temps, comme prisonniers de certaines valeurs et de certains réflexes qui déterminent par avance ce qu'ils sont supposés avoir comme réaction - en regard des contextes de vie - lorsque leur fils ou leur fille (surtout) leur annonce vouloir se marier avec quelqu'un qui ne fait pas partie des conjoints socialement acceptables. A ce titre, on notera aussi que l'opposition au mariage est plus souvent le fait des familles libanaises que palestiniennes, et que c'est presque toujours le cas lorsqu'une question identitaire est en jeu (appartenance communautaire, nationale, religieuse) ${ }^{30}$.

Au cœur de ce processus de contrôle sur l'identité du groupe, il y a la figure du père qui, dans de nombreux scénarii de mariage, joue un rôle central par l'accord qu'il donne, après des mois ou années de refus. Tout se passe à la manière d'un rituel composé de séquences précises : la déclaration de la fille (plus rarement du fils), le net refus du père, l'insistance de la fille (ou du fils) par divers procédés (dont l'enlèvement constitue un des extrêmes) et, enfin, la résolution par l'accord ou par la rupture, ultime soubresaut visant à emporter la décision par l'instauration d'un rapport de forces qui trouve sa résolution par la réconciliation dans un délai variable.

La dimension agie qu'adoptent les acteurs au sein de ce processus illustre bien l'importance du phénomène d'appartenance au groupe. Celui-ci est présent en tant que famille d'abord, mais plus fondamentalement en tant que lignage qui «prend » ou «donne » en mariage, suivant le sexe des enfants. Le groupe est présent ou plutôt omniprésent en tant qu'environnement social. Il définit des normes de vie commune, des règles de conduite et des valeurs. L'ombre des communautés est évidemment importante à ce niveau mais, du côté palestinien, la dimension collective infra-nationale (notamment villageoise) est également prégnante dans les camps ou dans certains quartiers avoisinants. Enfin, le groupe palestinien développe une appartenance nationale alors que, pour les Libanais, l'appartenance collective reste autrement plus éparse, précisément à cause de l'institutionnalisation des divisions du collectif entre communautés. C’est dire combien est important pour les différents « groupes » concernés le

29. En référence à la double dimension inhérente à l'action sociale dans la sociologie dispositionnaliste de Bourdieu. Voir Bourdieu P., Le Sens pratique, Paris, Minuit, 1980.

30. Par opposition aux questions plus matérielles (manque de ressources, second mariage, logement trop éloigné de ceux des parents de la femme, etc.) plus courantes chez les Palestiniens. 
fait de développer, à travers le mariage, une relation privilégiée au sens où, par cet acte, l'autre devient un allié par apparentement, c'est-à-dire devient membre du lignage, avec les droits et devoirs que cela comporte ${ }^{31}$.

Cet enjeu identitaire pour le groupe est donc loin d'être anodin et il se voit soumis à des règles écrites (les codes de statut personnel), non écrites, voire non dites (les codes d'honneur, normes de certaines communautés comme les druzes, ou croyances populaires propres à certains milieux sociaux). Ces règles sont pourtant connues de leurs membres et sont donc transgressées en connaissance de cause. On a vu qu'en cas de passage à l'acte (union matrimoniale) sans consentement parental ou après avoir forcé la main aux parents, la rupture familiale devient manifeste (parents absents lors de la cérémonie de mariage, rupture temporaire des relations entre parents et enfants) et dévoile le caractère quasi sacré de la parenté.

Cette sacralisation de la parenté est un phénomène plus caractéristique des sociétés traditionnelles, dans lesquelles statut et identité sociale sont transmis par la parenté ${ }^{32}$. L'existence de législations comme le code de statut personnel pour chacune des communautés libanaises témoigne de l'importance que revêtent la famille et la parenté dans ce dispositif. Dès lors, la transmission de la parenté est un enjeu identitaire de taille : au Liban, elle engage aussi bien l'appartenance communautaire que l'appartenance nationale. C'est dire que ces deux vecteurs identitaires se réfractent au sein des familles et redoublent parfois les effets transgressifs dans les cas d'unions sur la frontière nationale entre Libanais et Palestiniens.

On a vu avec l'exemple des mariages libano-palestiniens que la transgression est affaire de perception bien plus que de règles claires et précises. Ces dernières sont plutôt la portion congrue que le cadre rigide que l'on pourrait y voir en lisant à la lettre les codes de statut personnel. Après un rappel du cadre sociopolitique des relations libano-palestiniennes au Liban, nous avons tenté de montrer que certaines variables structuraient les rapports d'intermariages dans le cas étudié. Ainsi, la confession, la géographie, le capital économique et l'histoire sont apparus comme des variables déterminantes mais qui doivent être combinées, afin d'éviter toute tentation monocausale et toute simplification dans l'explication du phénomène. Les extraits de témoignages des couples libano-palestiniens ont permis de faire ressortir trois types de transgressions en mettant l'accent sur la perception des acteurs : la non-transgression, la transgression à impact réel et la transgression à rupture. Pour chacune de ces catégories, nous avons montré combien les acteurs opèrent des arrangements, combien aussi certains blocages fondés sur des croyances ou

31. Ghasarian C., Introduction à l'étude de la parenté, Paris, Le Seuil, 1996.

32 . Ibid., p. 11. 
perception de l'Autre (en tant que chiite, en tant que Palestinien, etc.) pouvaient constituer un frein à l'entreprise matrimoniale, et cela indépendamment d'une quelconque " gravité » avérée de la transgression commise.

En somme, ce travail sur les mariages entre deux groupes nationaux tend à minimiser l'importance réelle ou objective des actes transgressifs au profit de la dimension subjective de ceux-ci. S'il semble clair que, dans le cas d'espèce, les réglementations sont sujettes à interprétations, voire qu'elles sont conçues pour être interprétées, il n'en ressort pas moins que l'acte transgressif est une catégorie subjective qu'il faut mettre en lien avec un cadre normatif et un contexte social, au sens large. Ce terrain de recherche libanais, où tout semble pouvoir se négocier, est évidemment illusoire : le temps a en réalité renforcé des clivages, ossifié des positions et des perceptions, durcit des frontières invisibles. Dans cette étude, il subsiste les cas de non-mariages dont on n'a qu'une très faible connaissance, ces unions défaites sans avoir jamais pu se concrétiser par le mariage, réduites à néant en raison de tensions, d'un contexte aussi, de changements enfin qui, ces dernières années, ont été particulièrement destructeurs pour le tissu social local, mettant à rude épreuve les sociabilités libano-palestiniennes, d'abord en raison du clivage idéologique national mais aussi en raison des violences et du sang qui a de nouveau coulé durant l'été 2007 au Nord-Liban lors des affrontements entre le groupe jihadiste Fatab al-Islam et l'armée libanaise. C'est une manière de dire, également, que toute frontière, comme toute conclusion de recherche, reste provisoire, sujettes à aléas, à renégociations. 